\title{
Cross-Layer Congestion Control in Wireless Ad Hoc Network
}

\author{
Ali Alomari \\ School of Science and Arts \\ Clark Atlanta University, Atlanta, GA, 30314.
}

\begin{abstract}
Congestion control is a critical issue in mobile ad-hoc networks. This paper reflects a cooperatively ideal strategy of cross-layer congestion control, routing and setting up ad hoc wireless networks. We first calculate the degree check and scheduling check using multi-commodity flowing variables and calculate reserve sharing in networks with secure wireless channels as a service expansion issue with these controls. By dual corrosion, the reserve sharing problem naturally decomposes into three sub-issues: congestion control, routing, and scheduling that interact through congestion price. The overall junction property of this procedure is demonstrated. We next prolong the dual system to handle networks with time-varying channels and adaptive multi-proportion devices. The constancy of this resultant system is traditional, and its performance is characterized by an ideal position system which has the best feasible proportion state at the link layer. We then take a broad view the results mentioned above of an overall model of queueing network served by a set of interdependent parallel servers with time-varying service abilities, which models many design issues in communication networks. We show that for a curved optimization issue where a subset of variables lies in a polytope and the rest in a curved set, the dual-based procedure remains stable and optimum when the check set is modulated by an irreducible finite-state Markov series. This paper so offering a step toward a systematic way to carry out the cross-layer design by "Layering as optimization corrosion" for time-varying channel models.
\end{abstract}

\section{Keywords}

Congestion control; Mobile Ad-Hoc Network; TCP-overwireless; Multi-hop wireless networks; Cross-layer design.

\section{INTRODUCTION}

The purpose of this papers is to discuss the cross-layer congestion control, the issue of congestion control and reserve sharing through scheduling \& routing over a multihop wireless ad hoc network. Conventionally, network protocols take a severely layered configuration \& appliance congestion control, routing and scheduling freely at different coats. Wireless spectrum is a rare reserve, and it is important to use the wireless channel efficiently. To achieve foremost high-end throughput and efficient reserve utilization, congestion control, directing, and scheduling should be mutually designed while the structural separation among them is preserved. The need for dual design through these three layers is motivated by three explanations. First, the wireless channel is a shared medium and interference-limited. Unlike in wired networks where each link is combined reserves with fixed capacities in ad hoc wireless networks, the link functions are flexible, and the dispute among links provide a basic check for reserve sharing [1]. Second, most directing schemes for ad hoc networks select paths that minimize hop count [5]. It implicitly predefines a route for any source-destination pair of a static network, independent of the pattern of traffic demand and dispute among links. It may result in congestion at some state while other states are under-utilized. If we want to use the spectrum of wireless extra efficient, then we must have to achieve many multiple patterns depends on the path of traffic need and dispute between different links. As we will see below, directing is then determined from the proportion and scheduling checks.

Finally, TCP congestion control procedures can be interpreted as distributed primal-dual procedures over the Internet to maximize aggregate utility. This set of work obliquely adopts a network where link capacities are fixed, and routes are prespecified. At this point, we prolong the original utility maximization formulation with proportion checks at nodes and additional checks on scheduling at the link layer.

We model the dispute relations between wireless links as a conflict chart [4]. This creation shows that which clusters of different links mutually interfere \& cannot be lively simultaneously. The realistic proportion state at this link layer is the curved hull of the corresponding proportion vectors of free sets of the conflict map. We introduce multi-commodity flow variables to calculate proportion check at the network layer and calculate reserve sharing in wireless ad hoc networks with fixed channel or single-proportion devices as a utility maximization issue with those checks. We then apply duality theory to decay the system issue vertically into congestion control sub issue and routing/scheduling sub-issue that interact through congestion prices. Based on this corrosion, a distributed subgradient procedure for combined congestion control, routing and scheduling are obtained and proved to tactic randomly near to the optimum of this system issue. This procedure motivates a combined design where the source adjusts its sending proportion according to the congestion price generated locally at the source end \& backpressure from the differential price of neighboring ends is used for optimum scheduling and routing. We next prolong the dual subgradient procedure to wireless ad hoc networks with time-varying channels and adaptive multi-proportion devices. The stability of this system is hence proved, and the performance of this system is considered on perfect refereeing system.

\section{RELATED WORK}

The work in [6], [8] provides a utility-based optimization basis for Internet congestion control. The same basis has been applied to study the congestion control over ad hoc wireless networks [2] [1]. In [1], the authors study combined congestion control and media access control for the ad hoc wireless network and calculate proportion sharing as a utility maximization issue with the checks that arise from dispute for 
channel access. This paper substantially prolongs [3] to include routing and to study the network with the timevarying channel and multi proportion devices.

In [9], the authors use multi-commodity flow variables to characterize the network capacity state for a wireless network with the time-varying station and propose a combined routing and power sharing policy to stabilize the system whenever the input proportions are within this capacity state. In [4], the authors study the impact of interference on multi-hop wireless network performance. They model wireless interference using the conflict chart and exhibits that we have an opportunity for attaining throughput increases by employing an interferenceaware routing protocol. We use the same construction to model the dispute relations among wireless links. In [3], [7], the authors use a similar model to study the issue of jointly routing the flows and arrangement the transmissions to determine the attainable proportions in multi-hop wireless networks. All these works emphasis on the interaction between the different link and network coats and try to describe the achievable proportion state at the network layer. We include the endwise transport layer and network practices congestion control to automatically search the available proportion state while enhancing some general objective for end users.

Our aim is to present a methodical tactic to cross-layer design not only to progress the performance but more prominently, to mark the interactions between different layers more transparent. Driven by the dualism exemplary of TCP or $\mathrm{AQM}$ which is a sample of horizontal corrosion via dualism corrosion, researchers have prolonged the utility maximization basis to provide a basic cross-layer design methodology.

\section{RESULT AND METHODS}

Study an ad hoc wireless network with a set $n$ of nodes and a set $l$ of reasonable links. These links are directed. However, we assume connectivity to be symmetric link $(j, i) \in l$ if and only if $(i, j) \in l$. We adopt a standing topology, and each link $l$ has a fixed finite capacity $\mathrm{cl}$ bits per second when active. We tacitly assume that the wireless station is secure or some underlying mechanism is used to cover the channel variation so that the wireless channel appears to have a fixed proportion. The wireless network is a distributed medium and interference limited where links cope with each other for special access to the channel. We will use the conflict chart to capture the dispute relations among links. The feasible proportion state at link layer is then a curved hull of the corresponding proportion vectors of independent sets of the conflict chart. We will further present multi-commodity flow variable which resembles the link capacities owed to the flows to different destinations to describe the proportion check at the network layer. The reserve sharing is then calculated as a utility maximization issue with schedulability and proportion checks.

We use $1 \in 1$ or node pair $(\mathrm{i}, \mathrm{j}) \in \mathrm{n} \times \mathrm{n}$ to denote a link. We also stack up the entries of any (TDI) to create a vector indicated by $\{t i, j\}$ or $t$. Assuming the network is shared by a set $\mathrm{S}$ of sources indexed by s. For notational easiness, we adopt that there is utmost one flow between any node and endpoint pair $[\mathrm{i}, \mathrm{k}] 2$, and uses or node pair $[\mathrm{i}, \mathrm{k}] \in \mathrm{S} \times \mathrm{D}$ to denote a network layer flow. Assume each base attains a value Us(xs) when it conveys at proportion xs packets per second. We predict that Us is continuously differentiable, increasing, and firmly concave. Our objective is to choose source proportions xs and allocated capacities ok me,

$$
\begin{aligned}
& r_{l}^{e}:=\left\{\begin{array}{c}
c_{l} \text { if } l \in e \\
0 \text { otherwise }
\end{array}\right. \\
&\left\{\prod:=\mathrm{r:} \mathrm{r} \sum_{e} a_{e} r^{e}, a_{e} \geq 0, \quad \sum_{e} a_{e}=1\right\} \\
& \qquad x_{i}^{k} \leq \sum_{j:(i, j) \in L} f_{i, j}^{k}-\sum_{j:(j, i) \in L} f_{j, i}^{k} i \in N, k \\
& \in D, i \neq k
\end{aligned}
$$

Source proportion and congestion value:
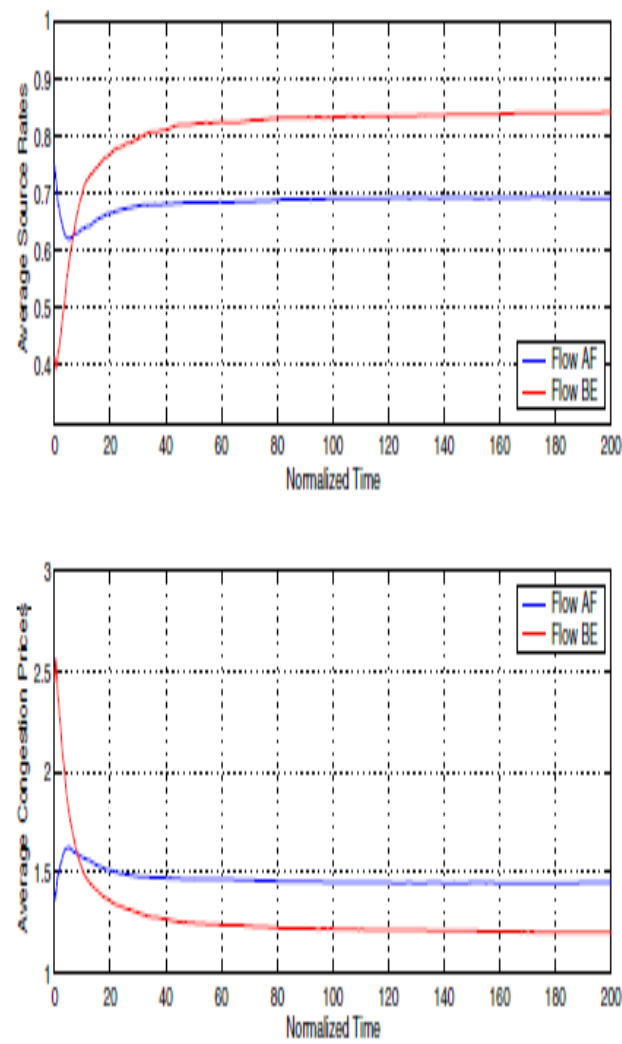

4. NUMERICAL EXAMPLE

NORMAL PROPORTIONS OF FLOWS $A F$ AND $B E$ THROUGH DIFFERENT LINKS WITH PROCEDURE THIRD

\begin{tabular}{|l|l|l|l|l|l|l|}
\hline Proportions & A & B & C & D & E & F \\
\hline A & 0 & 0.330 & 0.361 & 0 & 0 & 0 \\
\hline B & 0 & 0 & 0 & 0.095 & 0 & 0.239 \\
\hline C & 0 & 0.006 & 0 & 0.232 & 0.123 & 0 \\
\hline D & 0 & 0 & 0 & 0 & 0.004 & 0.323 \\
\hline
\end{tabular}




\begin{tabular}{|l|l|l|l|l|l|l|}
\hline E & 0 & 0 & 0 & 0 & 0 & 0.127 \\
\hline F & 0 & 0 & 0 & 0 & 0 & 0 \\
\hline
\end{tabular}

\begin{tabular}{|l|l|l|l|l|l|l|}
\hline Proportions & A & B & C & D & E & F \\
\hline A & 0 & 0 & 0.053 & 0 & 0 & 0 \\
\hline B & & & & & & \\
\hline C & 0.053 & 0 & 0.128 & 0.523 & 0 & 0.144 \\
\hline D & & 0 & 0 & 0.010 & 0.169 & 0 \\
\hline E & 0 & 0 & 0 & 0 & 0.498 & 0.035 \\
& & & & & & \\
\hline F & 0 & 0 & 0 & 0 & 0 & 0 \\
& & & & & & \\
\hline
\end{tabular}

Our simulation outcomes have long-established the assumptions from Theorem 5 and 6 , which say that the normal source proportions and congestion prices tactic the optimum of an ideal system with the best possible proportion state at the link layer, and that Procedure 3 can have been seen as a distributed procedure to solve this ideal system issue. We also have seen that the distributed scheduling procedure works well with the time-varying station, and the station variations, in fact, help mitigate the overall system's degradation due to suboptimum design in one layer.

\section{FUTURE WORK}

Additional research steps halting out of this research paper include the following. First, unique features in our procedure for practical implementations need to be further leveraged. Second, we will prolong the results to networks with more general interference models and node mobility. Third, scheduling issue is always a challenging issue for ad hoc network, and continued exploration of dispersed scheduling protocols will further enhance the performance increase from cross-layer strategy connecting different link layer. Next, we will properly enumerate the interesting observation that channel variations, in fact, help mitigate the overall system's degradation due to suboptimum design in one layer.

\section{CONCLUSION}

We have presented a model for the combined design of congestion control, routing and scheduling for ad hoc wireless networks by prolonging the basis of network utility maximization and applying dual-based corrosions. We calculate reserve sharing in the network with fixed wireless channels or single-proportion wireless devices as a utility maximization issue with schedulability and proportion checks arising from dispute for the wireless channel. By dual corrosion, we derive a subgradient procedure that is not only distributed spatially but more interestingly, decomposes the system issue vertically into three protocol layers where congestion control, routing, and scheduling solve the network utility maximization problem. We also prolong the dual procedure to handle the network with the time-varying channel and adaptive multi-proportion devices, and unpredictable show that, despite stochastic channel variation, it solves an ideal reference system issue which has the best feasible proportion state at the link layer.

Dualism procedures for curved optimization designs of widespread network utility expansion have found numerous applications recently for both stochastic \& deterministic level models. We demonstration that, for a large class of such curved optimization issues, stability and average performance are not affected by channel level stochastic models.It provides a general technique to carry out optimization based network designs in a time diverse environment.

\section{REFERENCES}

[1] L. Chen, S. Low and J. Doyle, Combined congestion control, and media access control design for ad hoc wireless networks, Proc. IEEE Infocom, 2005.

[2] M. Chiang, Balancing transport and physical layers in wireless multihop networks: jointly optimum congestion control and power control, IEEE J. Sel. Area Comm., vol. 23, no. 1, pp. 104-116, Jan. 2005.

[3] B. Hajek and G. Sasaki, Link Scheduling in polynomial time, IEEE Trans. Information Theory, 34:910-917, Sept. 1988.

[4] K. Jain, J. Padhye, V. N. Padmanabhan, and L. Qiu, Impact of interference on multi-hop wireless network performance, Proc. ACMMobicom, 2003.

[5] D. B. Johnson and D. A. Maltz, Dynamic source routing in ad-hoc wireless networks, Mobile Computing, (Eds. T. Imielinski and H. Korth), Kluwer Academic Publishers, 1996.

[6] F. P. Kelly, A. K. Maulloo and D. K. H. Tan, Proportion control for communication networks: Shadow prices, proportional fairness, and stability, Journal of Operations Research Society, 49(3):237-252, March 1998.

[7] M. Kodialam and T. Nandagopal, characterizing attainable proportions in multi-hop wireless networks: The combined routing and scheduling issue, Proc. ACM Mobicom, September 2003.

[8] S. Kunniyur and R. Srikant, End-to-end congestion control schemes: Utility functions, random losses and ECN marks, IEEE/ACM Transactions on Networking, 11(5):689-702, October 2003

[9] M. Neely, E. Modiano and C. Rohrs, Dynamic power sharing and routing for time-varying wireless networks Proc. IEEE Infocom, 2003. Journal version, IEEE J. Sel. Area Comm., 23(1):89-103, 2005. 